\title{
Österreichs Statistik und die Chancen der Informationsgesellschaft
}

\author{
Rainer Münz \\ Humboldt-Universität, Berlin
}

Österreichs Statistik wird selbständig. Daten zu sammeln, zu administrieren und auszuwerten, ist damit nicht mehr ein Akt und eine Aufgabe der öffentlichlichen Verwaltung, sondern wird zu einer Dienstleistung. Die bislang amtliche Statistik muß in Zukunft aufgrund von Aufträgen des Bundes, der Länder, einzelner Ressorts oder privater Interessenten tätig werden. Sie kann und wird dies nur tun können, soweit die öffentlichen und privaten Auftraggeber dies auch finanzieren.

Viele Mitarbeiter des zur Jahreswende 1999/2000 aus der Bundesverwaltung ausgegliederten Österreichischen Statistischen Zentralamts (ÖSTAT) erfüllt diese neue Autonomie mit Sorge. Sie sehen bloß das Ende einer 170-jährigen Geschichte, die mit der Gründung der k.k. Statistischen Zentralkommission im Jahr 1829 begann. Aber kaum jemand sieht die Chancen, ab nun privatwirtschaftlich agieren zu können. Ähnlich erging und ergeht es in Österreich den (Ex-)Beamten und Bediensteten von Bundesbahn, Post, Telekom, Energieversorgungsunternehmen, ehemals verstaatlichten Banken etc. Sie alle verlassen - mehr gezwungenermaßen als freiwillig - den geschützten Sektor, müssen umdenken und sich von lieb gewordenen Gewohnheiten verabschieden.

Bislang agierten die genannten Betriebe aus der Position des staatlich garantierten oder faktischen Spartenmonopols. Tarife, Personalentscheidungen und grundlegende Investitionsvorhaben waren politisch und durch bürokratische Logik bestimmt, aber keineswegs immer betriebswirtschaftlich motiviert. Zugleich fehlte es den halbstaatlichen Monopolbetrieben und ihren Mitarbeitern vielfach an der nötigen Kundenorientierung. Seitdem die genannten Betriebe zunehmend aus der Verantwortung des öffentlichen Sektors entlassen werden, zu börsennotierten Unternehmen mutieren und teilweise auch internationalem Wettbewerb ausgesetzt sind, verändern sich entscheidende Parameter. Strom und Telefon werden billiger, die Behandlung der Kunden von Mitarbeitern durch Bahn, Post und Telekom freundlicher, die Effizienz größer.

Vor der selben Herausforderung steht nun die bislang amtliche Statistik. Sie hat nach der Ausgliederung allerdings keine „Stunde Null“ vor sich. Es gibt auch in Zukunft eine ganze Reihe bundesgesetzlich angeordneter und folglich aus dem Staatshaushalt zu finanzierender Statistiken. Dies wird auf absehbare Zeit das „Kerngeschäft“ bleiben. Daneben „erbt“ Österreichs Statistik vom ÖSTAT den reichsten und vielfältigsten Datensatz des Landes. Diesen gilt es in Zukunft wesentlich systematischer, zielstrebiger und marktnäher zu nutzen. Mit Daten, die auch dem ÖSTAT zur Verfügung standen oder sogar von ihm gesammelt wurden, waren Österreichs Wirtschafts- und Sozialforscher (z.B. WIFO, IHS, Synthesis) schon bisher in der Lage, gegen Honorar Forschungsprojekte durchzuführen und Expertisen zu erstellen; also Geld zu verdienen. Dem ÖSTAT selbst fehlte es dazu sowohl an personeller Kapazität als auch an Reputation und Forschungsorientierung. Damit ist auch das Problem des Image und der Kommunikation angesprochen. Österreichs Statistik braucht ein innovatives, freundliches Erscheinungsbild: von der Vistenkarte über die Homepage bis zu Publikationen und Presseaussendungen. Und die Statistik muß sich 
einen Platz als Marktführer für bestimmte Dienstleistungen in der Informationsgesellschaft erarbeiten.

Wenn dies gelingt, dann eröffnet sich ein weiteres Geschäftsfeld im Bereich der Markt-, Konsum- und Meinungsforschung. Neben den laufenden Mikro-Zensuserhebungen böten sich für diese Zwecke auch die Erhebungen der Konsumgewohnheiten an, die bisher zur Berechnung der Verbrauchpreisindizes durchgeführt wurden. Auch neue, kommerziell nutzbare Erhebungen sind denkbar. Dabei gelte es, den eingespielten Interviewerstab des ÖSTAT zu nutzen.

Als weiteres Geschäftsfeld sollte sich die Statistik dem Bereich der laufenden Sozialberichterstattung und der Politikberatung erschließen. Schon bisher waren Mitarbeiterinnen und Mitarbeiter des ÖSTAT an der Erstellung von Familien-, Frauen-, Sozial- und Gesundheitsberichten beteiligt - entweder nebenberuflich oder per Amtshilfe.

In Zukunft könnte dies zu einer regulären Dienstleistung einer stärker privatwirtschaftlich agierenden Statistik werden. Da die Erstellung solcher Berichte seit einiger Zeit öffentlich ausgeschrieben wird, kann sich die Statistik in Zukunft auf die Expertise ihrer Mitarbeiter stützen und konkurrenzfähige Angebote erstellen.

Weitere potentielle Geschäftsfelder liegen außerhalb Österreichs. Sobald der Umbau von einem Amt zu einem effizienten Dienstleistungsunternehmen gelungen ist, kann sich Österreichs Statistik allein oder in Kooperation mit ausländischen Partnern um EUweit ausgeschriebene nationale Aufträge in anderen Ländern sowie um Aufträge der EUKommission bewerben. Außerdem besteht die Chance, die bei der Ausgliederung aus der Bundesverwaltung gewonnenen Erfahrungen auch anderswo nutzbringend zu verwerten: z.B. durch Beratung beim anstehenden Umbau der statistischen Ämter und Dienste anderer EU-Staaten oder unserer östlichen Nachbarländer. Dazu nötige personelle und institutionelle Kontakte sind bereits jetzt vorhanden.

Im Informationszeitalter, als das wir das ausgehende 20. und anbrechende 21. Jahrhundert bisweilen bezeichnen, saß und sitzt die bislang amtliche Statistik auf Daten, aus denen sich erheblicher wirtschaftlicher Nutzen ziehen läßt. In den USA ist die kommerzielle Nutzung von Informationen aus Volkszählungen, laufender Bevölkerungs- und Wirtschaftsstatistik sowie der Wanderungsstatistik längst ein etablierter Geschäftszweig mit wachsenden Umsätzen und einer steigenden Zahl von Arbeitsplätzen. In Europa stehen wir diesbezüglich noch am Anfang. Wenn Österreichs Statistiker lernen, die Ausgliederung als Chance zu sehen, können sie auf dem boomenden Zukunftsmarkt der Informationsbeschaffung und Informationsverarbeitung nur gewinnen.

Rainer Münz war geschäftsführender Direktor des Instituts für Demographie der Österreichischen Akademie der Wissenschaften. Seit 1992 ist er ordentlicher Professor für Bevölkerungswissenschaft an der Berliner Humboldt-Universität. 
Adresse des Autors:

O.Univ.-Prof. Dr. Rainer Münz

Lehrstuhl Bevölkerungswissenschaft

Humboldt-Universität

D-10099 Berlin, Deutschland

Tel.: 0049-30-2093 1918

Fax: 0049-30-2093 1432

Email: rainer.muenz@sowi.hu-berlin.de

Online: www.demographie.de 
